\title{
Energy-Aware Gas Sensing Using Wireless Sensor Networks
}

\author{
Andrey Somov ${ }^{1}$, Alexander Baranov², Alexey Savkin², Mikhail Ivanov², \\ Lucia Calliari ${ }^{3}$, Roberto Passerone ${ }^{4}$, Evgeny Karpov ${ }^{5}$, and Alexey Suchkov ${ }^{5}$ \\ ${ }^{1}$ CREATE-NET, Trento, Italy \\ andrey.somov@create-net.org \\ ${ }^{2}$ «MATI» - Russian State Technological University, Moscow, Russia \\ radio@mati.ru \\ ${ }^{3}$ FBK-Centro Materiali e Microsistemi, Trento, Italy \\ calliariafbk. eu \\ ${ }^{4}$ University of Trento, Trento, Italy \\ roberto.passeroneddisi.unitn. it \\ ${ }^{5}$ Scientific and Technical Center of Measuring Gas Sensors, Lyubertsy, \\ Moscow Region, Russia \\ karpovef@trancom.ru
}

\begin{abstract}
Wireless Sensor Networks (WSN) have recently been applied in various monitoring applications including hazardous gases detection. However, being a major power consumer of a sensor node, off-the-shelf gas sensors significantly constrain its lifetime. In this paper we present a WSN for hazardous gases detection with a special focus on the power consumption of the sensor node. The sensor node is designed on the basis of a planar catalytic sensor with improved power consumption characteristics. The power supply of the node is divided into digital and analogue parts. This is done to guarantee digital remote control of the device even when the analogue power source has already been depleted by the sensing circuit. In addition, we propose a differential gas measurement approach along with specific heating pulses for the sensor to secure substantial energy saving. The resulting average power consumption is 1.45 and $2.64 \mathrm{~mW}$ for the gas sensor and the sensor node respectively. With our techniques, the sensor node lifetime improves from 187 days up to 641 days.
\end{abstract}

Keywords: wireless sensor network, catalytic sensor, remote sensing, gas detection, dual power supply.

\section{Introduction}

A Wireless Sensor Network (WSN) is a distributed collection of sensor nodes which measure ambient conditions, store and/or communicate the data over the network by wireless means. A sensor node consists of four main blocks: a radio transceiver, a sensor, a processing unit (with memory), and a power supply. Depending on the application, the node may contain extra hardware parts. 
WSNs have been applied in multiple areas [1] including hazardous gas detection [2]. Due to their limited capacity, the energy buffers of a sensor node, i.e. batteries and/or supercapacitors, must be used efficiently. The on board radio chip is typically the most power-hungry component of the sensor node [3]. However, the WSNs aimed at early hazardous gases detection adopt sensors (spectroscopic, catalytic, semiconductor) which may consume currents of up to $800 \mathrm{~mA} \mathrm{[4],} \mathrm{[5],} \mathrm{[6],} \mathrm{[7].} \mathrm{There} \mathrm{are}$ some commercial off-the-shelf systems available on the market with high power consumption [8] and limited up to 14-hours [9] life time cycle. The sensor node lifetime problem in this case seems to be of vital importance.

To overcome this problem, several alternative platforms with energy efficient on board sensors were proposed [10], [11]. These platforms do not provide high accuracy and sensitivity though. Besides, their long response time equal to approximately 5 minutes [10] does not comply with safety standards [12]. Other recently proposed approaches to address the lifetime problem (the state-of-the-art systems claim approximately 187 days of life cycle operation [2]) in the Gas WSN (GWSN) domain include the application of energy scavenging technology [8], [13], the employment of a pulse heating profile for the sensing unit [2], and the establishment of a trade-off between the sensing and communication units of the node [14].

In fact, high power consumption of the gas sensor may result in situations when the sensing may fully deplete the energy storage with no chance to transmit an alarm message to a network coordinator. In this case the late detection of hazardous gas may lead to human victims and pecuniary losses. In order to prevent situations of this kind, it is highly desirable to secure the communication unit from de-energizing.

In this work we present a WSN for early hazardous gas detection with a special focus on lifetime of the sensor nodes. Apart from the sensor nodes, the WSN includes three units: a relay node, a network coordinator, and a wireless actuator. To avoid the deenergizing of the sensor node communication unit, we employ a dual (digital and analogue) power supply. The digital one supplies processing and communication units, the analogue the sensing one. More importantly, we propose and implement a number of techniques aimed at improving the long-term operation of the sensor node. First, we shift from typical catalytic sensors to sensors made using planar design. Second, instead of a gas measurement circuit based on a Wheatstone bridge with two sensors, we propose a differential technique which requires one sensor and a sophisticated heating pulse. The range of wireless transmission is extended using the relay nodes, which support communication between the sensor node and the network coordinator. Whenever a hazardous gas is detected in the atmosphere, the network coordinator alarms an operator by the GSM/GPRS or Ethernet network and/or controls a gas emission source by the wireless actuator.

The paper is organized as follows: we introduce the reader to developed WSN in Section 2. After that we describe the catalytic sensor design and energy efficient gas measurement in Section 3. The sensor node design and the problem associated with its long-term operation are presented in Section 4. The network operation is shown in Section 5. Finally, we discuss the results and conclude in Section 6. 


\section{WSN Overview}

The developed WSN for hazardous gases detection (see Figure 1) consists of four units: a sensor node, a relay node, a wireless actuator, and a network coordinator. Depending on the gas monitoring scenario, the WSN may include various number of units of each type. Since the communication range of the TG-ETRX35x (hereafter TG-ETRX3, or ETRX3) employed transceiver is only around $20 \mathrm{~m}$, we use relay nodes. The relay node consists of a TG-ETRX3 transceiver and a power supply. Data routing is performed by the firmware of the transceiver. The firmware forms a cluster topology with a maximum number of hops up to 30 .

The network coordinator is the main unit of the WSN. It supports the network operation by wireless communication based on the IEEE802.15.4 standard and the ZigBee specification. The network coordinator is also responsible for alerting a network operator or an emergency service using the Ethernet network or sending a SMS using a GSM/GPRS modem. In fact, upon receiving the alert message from the sensor node, the network coordinator can perform the first counter actions by deactivating the source of gas emission via the wireless actuator.

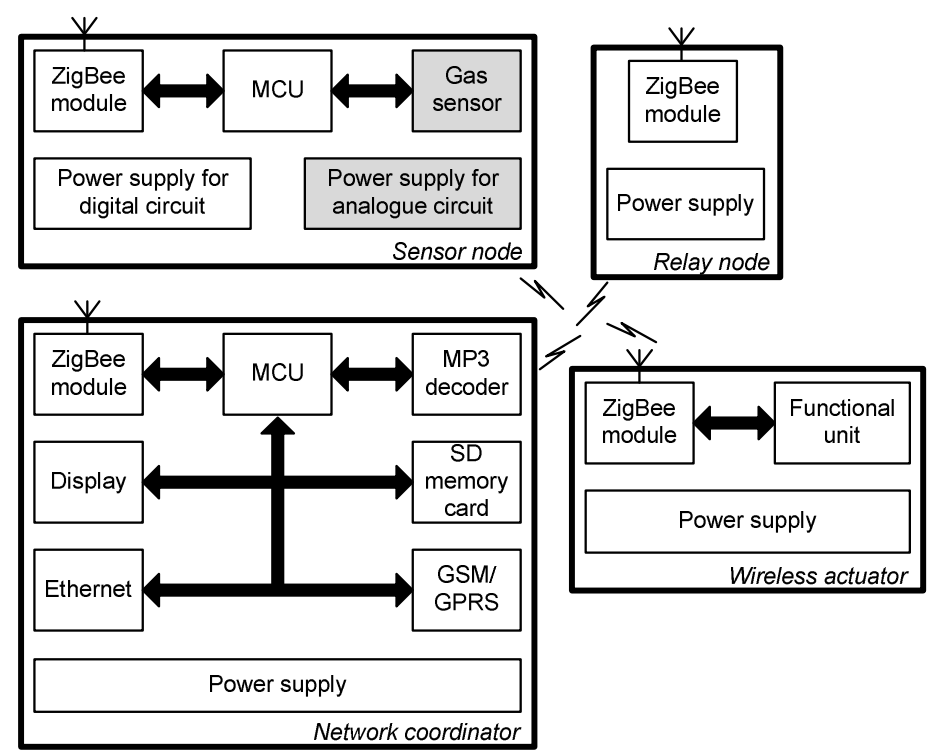

Fig. 1. Block diagram of the wireless sensor network for hazardous gases monitoring (sensor node, relay node, network coordinator, and wireless actuator)

Wireless communication between the network coordinator and the sensor node/wireless actuator can be implemented via the relay node(s) to extend the WSN coverage. The network itself is automatically organized by the ZigBee modules installed on each unit.

The goals of the sensor node are environmental monitoring, early detection of targeted hazardous gas, and alerting of the network coordinator. Successful monitoring, 
however, requires the availability of significant energy resources. In the following sections, we demonstrate how we reduce the power consumption of the sensor node.

\section{Catalytic Sensor for the Sensor Node}

This section is motivated by the need of efficient catalytic gas sensor with extremely low average energy consumption required for wireless sensor nodes powered by portable batteries. In practice, a typical on board gas sensor consumes more power than a microcontroller (MCU) or a transceiver. Table 1 shows the power consumption of some off-the-shelf components used in a sensor node design. Since the current drawn by the gas sensors changes during their operation, we report their consumption in terms of power $(\mathrm{mW})$. For the other components, which can operate under different voltage levels, we report the current consumption $(\mathrm{mA})$ one. The data are reported from the web pages of the respective manufacturers.

Table 1. Power consumption of some off-the-shelf components used in sensor node design

\begin{tabular}{|c|c|c|c|}
\hline & Type & Manufacturer & $\begin{array}{l}\text { Power }(\mathrm{mW}) \text { or } \\
\text { current consumption }(\mathrm{mA})\end{array}$ \\
\hline \multirow{8}{*}{ 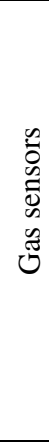 } & DTK-2 & NTC-IGD, Russia & $120 \mathrm{~mW}$ \\
\hline & SGS-21XX & Delta, Russia & $200 \mathrm{~mW}$ \\
\hline & $\begin{array}{l}\text { TGS2610 } \\
\text { semiconductor }\end{array}$ & FIGARO, Japan & $280 \mathrm{~mW}$ \\
\hline & NAP-66A & Nemoto, Japan & $360 \mathrm{~mW}$ \\
\hline & catalytic & & \\
\hline & MQ-4 & Hanwei & $750 \mathrm{~mW}$ \\
\hline & & Electronics, China & \\
\hline & $\begin{array}{l}\text { NLK series } \\
\text { laser spectroscop- } \\
\text { ic }\end{array}$ & $\begin{array}{l}\text { NTT Electronics, } \\
\text { Japan }\end{array}$ & $800 \mathrm{~mA}$ \\
\hline \multirow{7}{*}{ 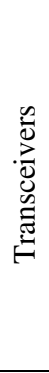 } & $\mathrm{CC} 2500$ & Texas & Tx: $21.2 \mathrm{~mA} @ 0 \mathrm{dBm}$ \\
\hline & & Instruments & $\mathrm{Rx}: 13.3 \mathrm{~mA}$ \\
\hline & CC2430 & Texas & Tx: $27 \mathrm{~mA}$, \\
\hline & & Instruments & $\mathrm{Rx}: 25 \mathrm{~mA}$ \\
\hline & ETRX35x & Telegesis & $\begin{array}{l}\text { Tx: } 31 \mathrm{~mA} @+3 \mathrm{dBm} \text {, } \\
\text { Rx: } 25 \mathrm{~mA} @ 12 \mathrm{MHz} \text { clock speed }\end{array}$ \\
\hline & TR1000 & RF Monolithics & Tx: $12 \mathrm{~mA} @ 0 \mathrm{dBm}, \mathrm{Rx}: 3.1 \mathrm{~mA}$ \\
\hline & $\begin{array}{l}\text { JN5148-001- } \\
\text { M00/03 }\end{array}$ & Jennic & $\begin{array}{l}\text { Tx: } 15 \mathrm{~mA} @+2.5 \mathrm{dBm} \text {, } \\
\text { Rx: } 17.5 \mathrm{~mA}\end{array}$ \\
\hline \multirow{4}{*}{ 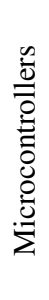 } & MSP430F247 & $\begin{array}{l}\text { Texas } \\
\text { Instruments }\end{array}$ & $\begin{array}{l}\text { Active mode: } 321 \mathrm{uA} @ 3 \mathrm{~V} / 1 \mathrm{MHz} \\
\text { Low power mode: } 1 \mathrm{uA} @ 3 \mathrm{~V} / 32768 \mathrm{~Hz}\end{array}$ \\
\hline & ATmega168P & Atmel & $\begin{array}{l}\text { Active mode: } 1.8 \mathrm{~mA} @ 3 \mathrm{~V} / 4 \mathrm{MHz} \\
\text { Power-save mode: } 0.9 \mathrm{uA} @ 3 \mathrm{~V} / 32 \mathrm{kHz}\end{array}$ \\
\hline & ATxmega32A4 & Atmel & $\begin{array}{l}\text { Active mode: } 1.1 \mathrm{~mA} @ 3 \mathrm{~V} / 2 \mathrm{MHz} \\
\text { Power-save mode: } 0.7 \mathrm{uA} @ 3 \mathrm{~V} / 32 \mathrm{kHz}\end{array}$ \\
\hline & $\mathrm{ADuC} 824$ & Analog Devices & $\begin{array}{l}\text { Active mode: } 3 \mathrm{~mA} @ 3 \mathrm{~V} / 1.5 \mathrm{MHz} \\
\text { Power-down mode: } 20 \mathrm{uA} @ 3 \mathrm{~V} / 32 \mathrm{kHz}\end{array}$ \\
\hline
\end{tabular}


For the wireless sensor node we have developed a planar catalytic sensor on gamma alumina membranes with a thickness of 30 um (Figure 2a). To avoid bending of the membrane during periodic heating (eventually leading to membrane failure) and to further decrease energy consumption, we use free wedge-shaped membranes without linking them to a rigid frame. Micro-heater patterns are formed by lithography on top of the membrane. Micro-heaters are deposited by magnetron sputtering of a platinum (Pt) target. The heated area is about 200x200 um (Figure 2b).

Micro-heaters are covered by porous gamma alumina oxide material that is used as catalyst support for catalytically active metals (mixture Pd and Pt). In order to impregnate the catalyst support by catalytic metal, salts of palladium chloride $\left(\mathrm{PdCl}_{2}\right)$ and platinum acid $\left(\mathrm{H}_{2} \mathrm{PtCl}_{6}\right)$ are used. The noble metal clusters are formed on the catalyst support after annealing.

Planar catalytic methane sensors were tested in the pulsed operating regime in order to measure dynamic parameters, to optimize the heating velocity and to minimize energy consumption.

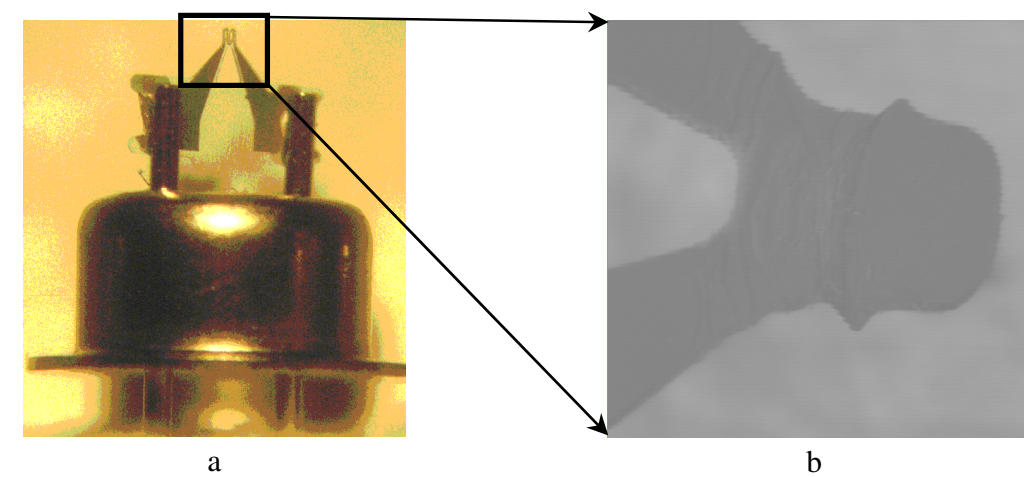

Fig. 2. a) Sensor picture (magnification 20X). The microheater is on the top. b) Electronic picture of microheater covered by catalyst (magnification 250X).

To reduce energy consumption during the measurements, we propose to avoid the Wheatstone bridge measuring circuit used in the majority of current systems [2], [13]. However, this leads to the need of a compensation method for the effect of environmental parameters (in particular, humidity and temperature) on the measurement results. For this reason, we have developed a differential method for measuring methane concentration using a single gas catalytic sensor with compensation for changes in ambient temperature, relative humidity and atmospheric pressure.

The method consists in measuring the difference between the sensor response at two different characteristic temperatures of catalysis: the beginning of the kinetic region $\left(\sim 200{ }^{\circ} \mathrm{C}\right)$ and the start of the external diffusion region $\left(\sim 450{ }^{\circ} \mathrm{C}\right)$. For this purpose, the sensor is heated by applying a 4-stage voltage pulse (Figure 3). Overall, during each cycle, the sensor is heated for about $250 \mathrm{~ms}$. 


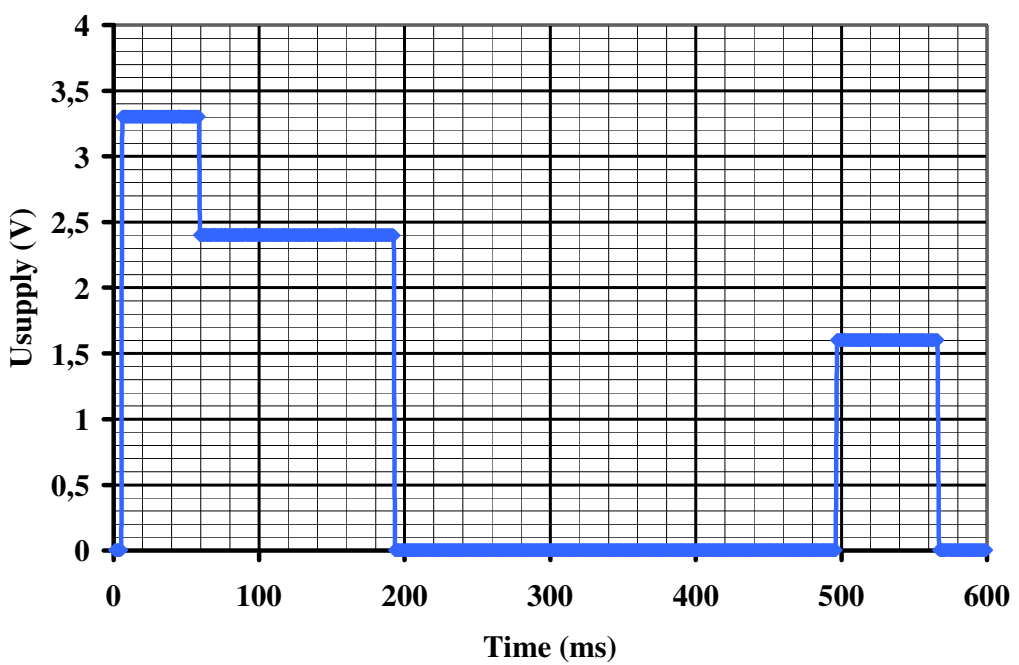

Fig. 3. Four-stage pulse of voltage applied to the sensor heater

The implementation of the differential method of measurement is performed by powering the sensor with a pulse, where the first and second pulses (afterburner for 55 $\mathrm{ms}$ and the support for $150 \mathrm{~ms}$ ) provide the sensor heating to the external diffusion region of catalysis and the partial evaporation of surface water $\left(\sim 450{ }^{\circ} \mathrm{C}\right)$. These stages are followed by a pause (third stage) during which the surface is not heated. The final voltage pulse (fourth stage) heats the sensor to the beginning of the kinetic region of catalysis $\left(\sim 200{ }^{\circ} \mathrm{C}\right)$. After this pulse, the element cools down to ambient temperature. Then, the cycle repeats after $30 \mathrm{~s}$.

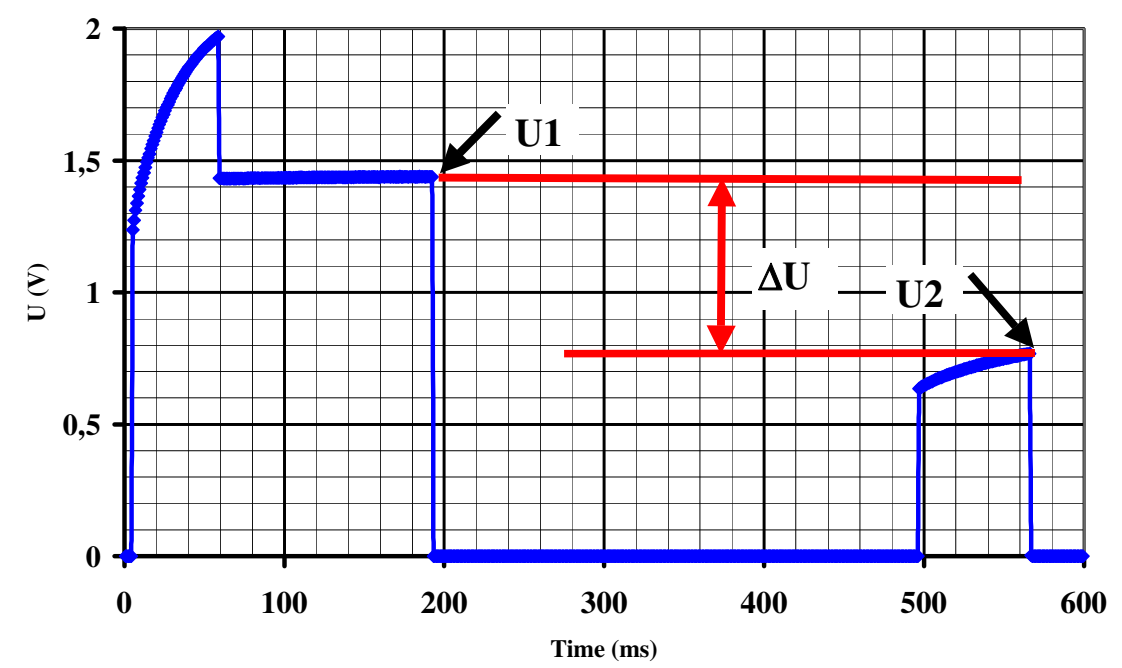

Fig. 4. Voltage across the sensor after application of the four-stage pulse. Output signal $\Delta U=U_{1}-U_{2}$. 
The output of the sensor, i.e., the voltage measured across the sensor surface, is sampled twice during a period. The first value, $U_{l}$, is the voltage at the end of the second stage of pulse heating (which heats the sensor up to $450{ }^{\circ} \mathrm{C}$ and lasts for $t_{l}=190 \mathrm{~ms}$ ). $U_{2}$ is the voltage at the end of the fourth stage of pulse heating (which heats the sensor up to $200{ }^{\circ} \mathrm{C}$ and lasts for $t_{2}=550 \mathrm{~ms}$ ). This is done to compensate the absorption of moisture on the sensor surface that occurs between two cycles, and influences the quality of the measurement. After the first two heating pulses, the water particles evaporate only partially, while much longer heating pulses would be required to guarantee full evaporation, immediately resulting in higher power consumption. The heating pulse duration have been defined to achieve an almost equal quantity of non evaporated water on the sensor's surface at $U_{1}$ and $U_{2}$ (see Figure 4). By taking the output of the sensor as the difference between $U_{l}$ and $U_{2}$, the influence of absorbed moisture is compensated.

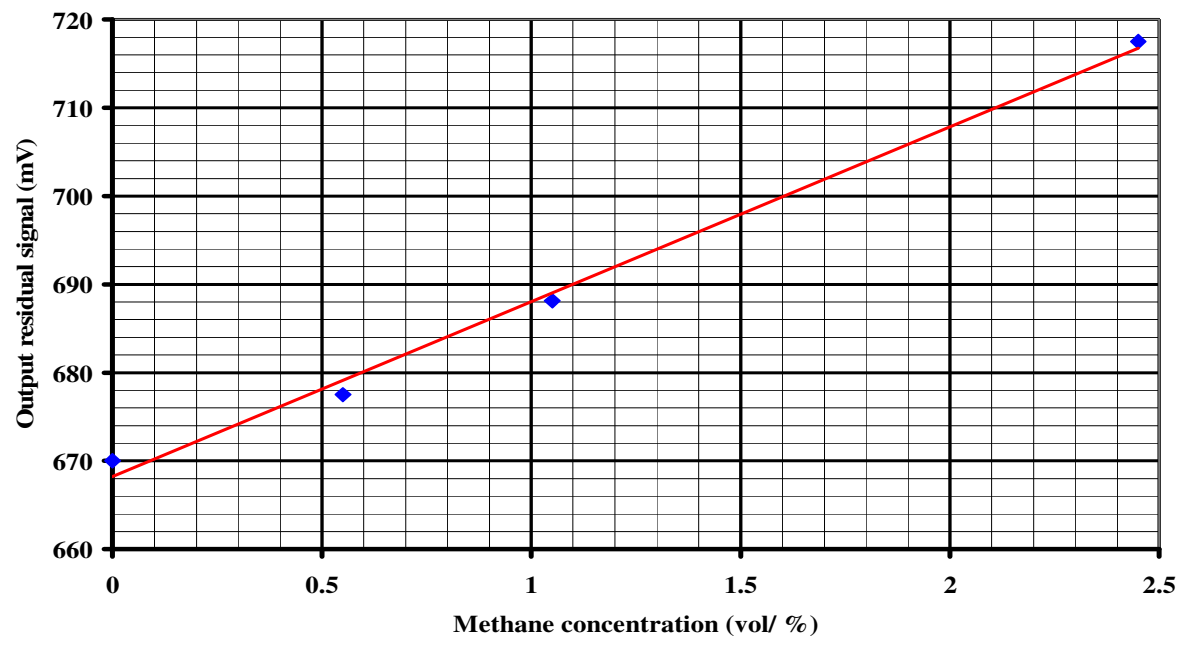

Fig. 5. Output signal of the sensor as a function of methane concentration

The dependence of the output signal of the sensor on the methane concentration is shown in Figure 5. The specific sensitivity of the sensor under test was $19.8 \mathrm{mV} /$ $\% \mathrm{CH}_{4}$. The absolute error of measurement of methane concentration was less than $0.1 \%$ volume fraction.

\section{Sensor Node Design}

The main purpose of wireless gas sensor node in the network is to perform methane measurement using the planar catalytic sensor described in Section 3 and to communicate the data to the network coordinator or activate the wireless actuator in the case of alarm situation. 
The wireless sensor node is based on the ATxmega32A4 microcontroller (MCU). This MCU has been chosen due to its low power consumption $(\sim 1 \mathrm{uA}$ in power-save mode), sufficient performance and high-precision 12-bit ADC and DAC. To provide communication across the WSN, we have used an ETRX357 ZigBee transceiver. It complies with the IEEE 802.15.4 standard, operates in the $2.4 \mathrm{GHz}$ ISM band and has a low sleep current $(\sim 0.7 \mathrm{uA})$. Both devices support a wide supply voltage range from $2.1 \mathrm{~V}$ to $3.6 \mathrm{~V}$.

A noteworthy feature of the sensor node is its use of a dual power supply for the digital and analogue circuits (see Figure 6). Each power supply is composed of batteries. The first battery (GB1) is composed of three C-sized alkaline cells and supplies the gas sensor system ('analogue circuit'). The second battery (GB2) is a AA-sized lithium-thionyl chloride $\left(\mathrm{Li}-\mathrm{SOCl}_{2}\right)$ cell with a nominal capacity of $2.6 \mathrm{Ah}$ and it powers the MCU and the transceiver ('digital circuit'). Such design prevents digital noise from entering the analogue circuit. In addition, it provides high reliability and long lifetime of the device because the average sensor power consumption exceeds the digital circuit consumption. Thereby, in case of a discharge of the first battery, the node does not disconnect from the WSN and can transmit alert messages.

Typically, the methane measurements are realized by a pair of an active and reference catalytic sensors embedded in a Wheatstone bridge. However, our processing technique allows us to use only one active catalytic sensor applied in a voltage divider. The circuit with one sensor has worse response (which, in fact, can be amplified by software gain), but provides significant energy savings.

The voltage across the gas sensor is provided by an ADP161 linear regulator. It is enabled by the MCU and adjusted via the DAC which uses the ADP161 output as a reference voltage. Thus, there is a feedback loop, which secures the sensor voltage from the voltage deviations of power supply caused by a constant battery discharge. In the other respects, the hardware design was implemented according to the manufacturer specifications.

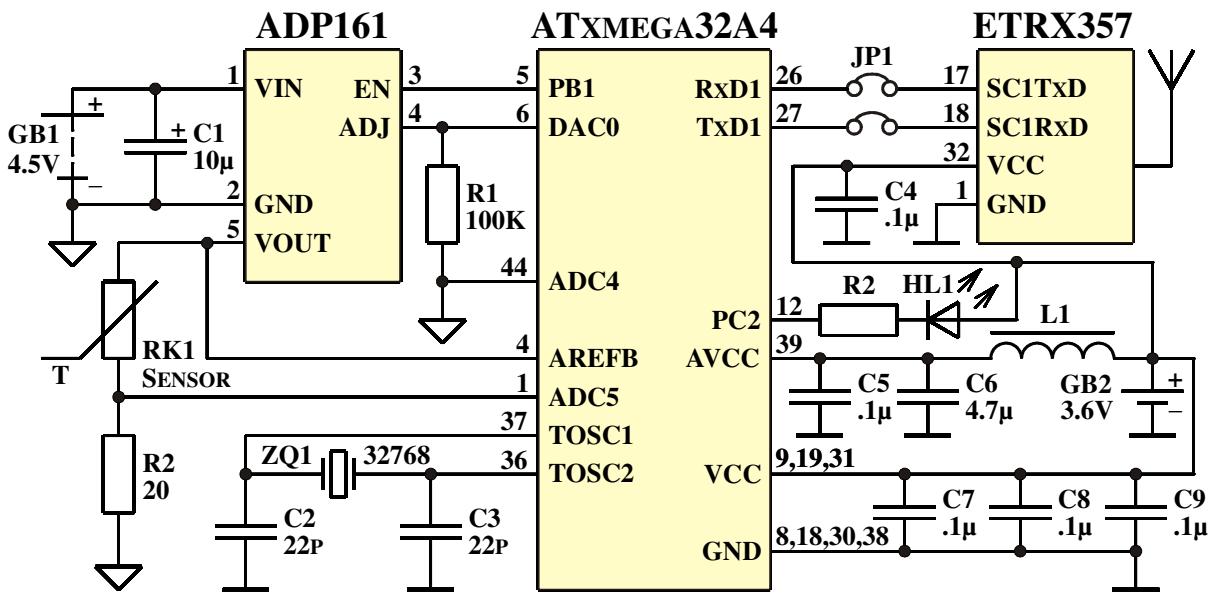

Fig. 6. Hardware architecture of the wireless gas sensor node 
The operation algorithm of the node is defined by the MCU firmware, written in C.

After power up, the MCU initializes its peripherals: the GPIO ports, the interrupt system, a real time counter (RTC) and the watchdog timer. The CPU clock frequency is set to $2 \mathrm{MHz}$, USART baud rate is $19200 \mathrm{bps}$, The DAC is set to low power mode. Afterwards, the MCU sends AT-style commands via the USART interface to the transceiver to configure it as a ZigBee End Device, join a WPAN and then switch the transceiver to sleep mode.

Thereafter, the MCU starts a cycle of measurement sequences. The sequences consist of several stages: measuring, data transmission to the network coordinator, and sleeping. At the measuring stage, the MCU enables heating of the gas sensor, acquires the sensor response by the ADC and processes the data. It should be observed that the ADC operates in a differential input mode with gain, where inputs ADC5 and ADC4 are exploited as a differential pair. The gain factor, which is used to amplify the signal before conversion, is 4 . The ADC clock frequency is $125 \mathrm{kHz}$ and uses an accurate internal $1.00 \mathrm{~V}$ reference voltage. In order to reduce possible ADC errors, the MCU acquires 16 samples and stores their mean value.

Figure 7 and Figure 8 show the current consumption of the sensor node with the Wheatstone bridge (with the catalytic bead sensors) circuit and with the differential (with the planar sensor) measurement technique (described in Section 2), respectively. Methane is used for the test measurements. For both approaches, a heating voltage of $2.8 \mathrm{~V}$ is applied during $700 \mathrm{~ms}$ to heat the sensor(s) up to the operation temperature $\left(450^{\circ} \mathrm{C}\right)$, but with a different profile.

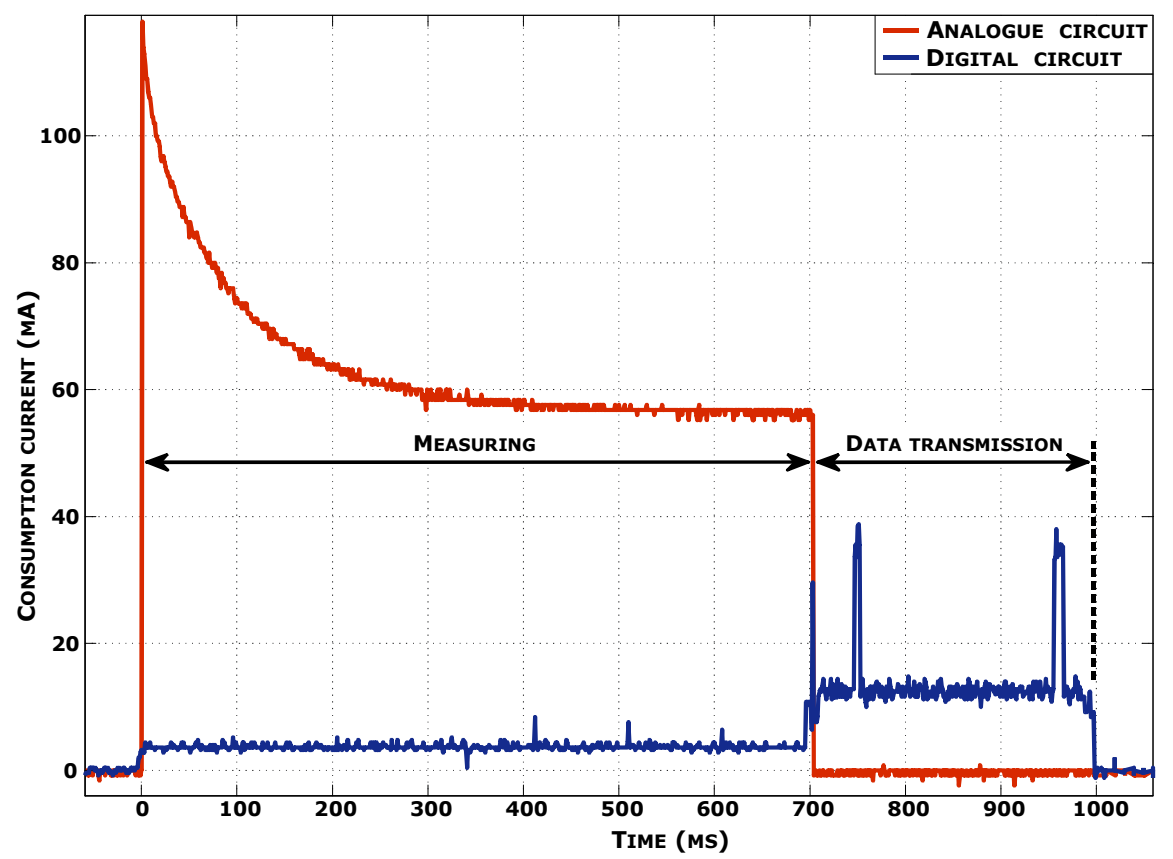

Fig. 7. Power consumption diagram for the gas sensor node with two catalytic bead sensors embedded in a Wheatstone bridge 
Just after measuring (in both cases), the MCU disables the linear regulator, the ADC and the DAC. Then, the MCU processes the gas sensor data, awakes the transceiver and sends the AT command to transmit a unicast to a sink node. Besides sensor data, the transmitted unicast contains battery charge information.

To ensure energy saving, the data transmission is performed only in case the gas concentration exceeds a threshold (warning threshold is $0.25 \%$, alarm threshold is 1 $\%)$. If the gas concentration does not exceed these thresholds, the sensor node sends unicasts every 5 minutes to inform of the correct status of operations.

To ensure successful transmission, the node waits for an acknowledgement. If unicast transmission fails, the node will repeat it twice. If all of them fail, the node will attempt to rejoin a WPAN every 60 seconds.

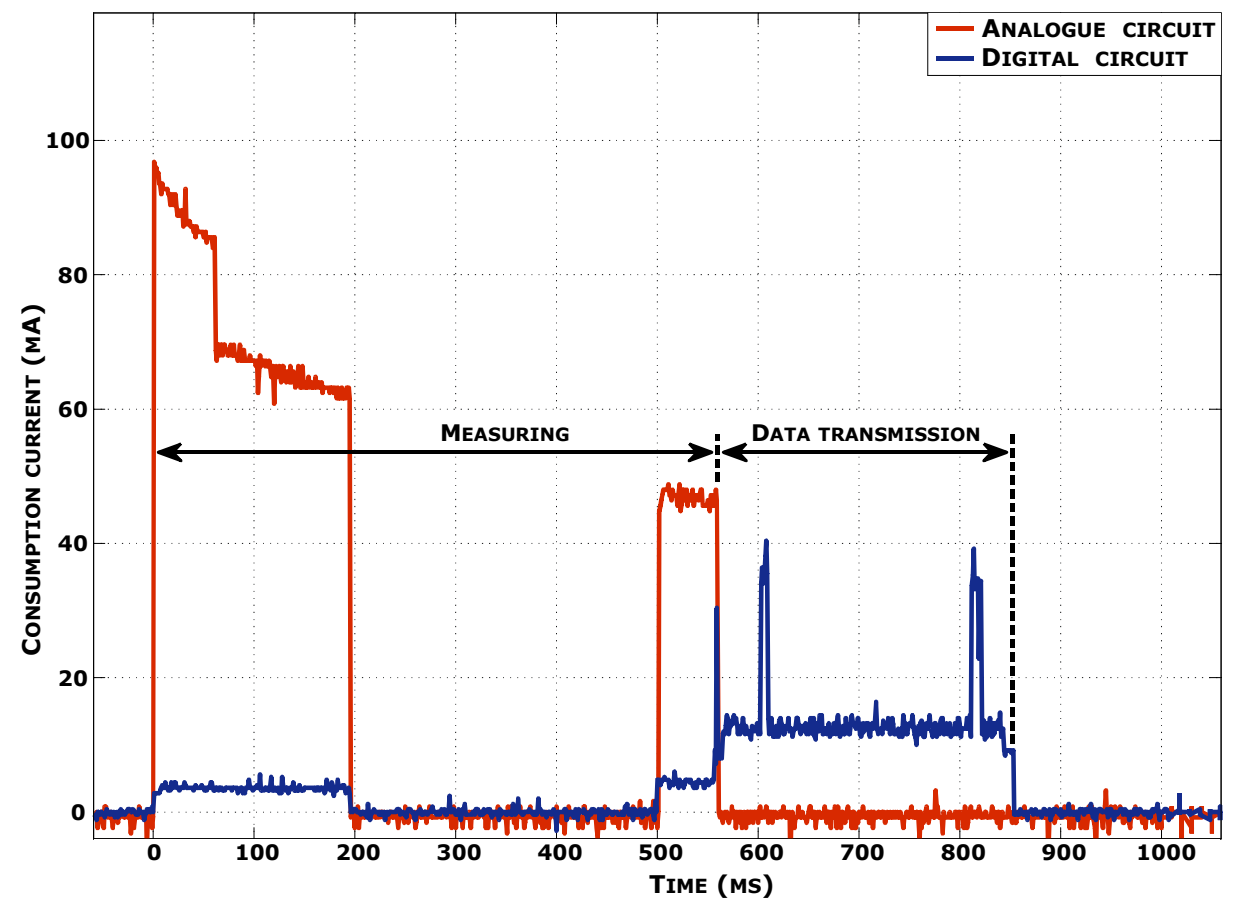

Fig. 8. Power consumption diagram for the wireless gas sensor node with a single catalytic planar gas sensor in a voltage divider

After successful transmission, the node immediately turns to power-save mode and stays in this mode for 30 seconds. The current consumption of the node in power-save mode is about $1.8 \mathrm{uA}$. All modules and clocks of the MCU are shut down except for the RTC, which is clocked by an external $32.768 \mathrm{kHz}$ crystal oscillator. The RTC interrupts are used to wake-up the MCU. After waking up, the measurement sequence is repeated.

The calculated values of the power consumption and estimation of battery lifetime are presented in Table 2 . The average power consumption, $P_{a v}$, is as follows: 


$$
P_{a v}=\frac{1}{T} \int_{0}^{T} u(t) \cdot i(t) d t
$$

where $u(t)$ is voltage, $i(t)$ is current, $T$ is the time period (30 s). It should be noted that the digital-to-analogue converter of the MCU operates during measurement. Therefore, the Wheatstone circuit results also in higher digital power consumption, since the converter is kept in the on state for longer time than with the differential technique. The obtained results demonstrate that the application of the differential measurement technique ensures the sensor node operation almost for two years.

Table 2. Average power consumption and lifetime estimation of the sensor node

\begin{tabular}{c|c|c|c|c}
\hline \multirow{2}{*}{$\begin{array}{c}\text { Sensor node } \\
\text { design }\end{array}$} & Parameter & $\begin{array}{c}\text { Communi- } \\
\text { cation every } \\
30 \mathrm{~s}\end{array}$ & $\begin{array}{c}\text { Communi- } \\
\text { cation every } \\
5 \mathrm{~min}\end{array}$ & $\begin{array}{c}\text { Analogue circuit } \\
\text { (sensors) }\end{array}$ \\
\hline \multirow{2}{*}{$\begin{array}{c}\text { with Wheat- } \\
\text { stone bridge }\end{array}$} & $P_{a v}, \mathrm{~mW}$ & 0.82 & 0.50 & $6.65(4.18)$ \\
\cline { 2 - 5 } & Life time, days & 476 & 781 & 225 \\
\hline $\begin{array}{c}\text { with single } \\
\text { sensor circuit }\end{array}$ & $P_{a v}, \mathrm{~mW}$ & 0.62 & 0.30 & $2.34(1.45)$ \\
\cline { 2 - 5 } & Life time, days & 629 & 1308 & 641 \\
\hline
\end{tabular}

The node has been housed into a sealed plastic container (Figure 9a,b). The catalytic gas sensor has explosion-proof construction. For status indication of the node, a LED was built in. Also, in the future the node will be supplemented by a buzzer to give an acoustic alert.

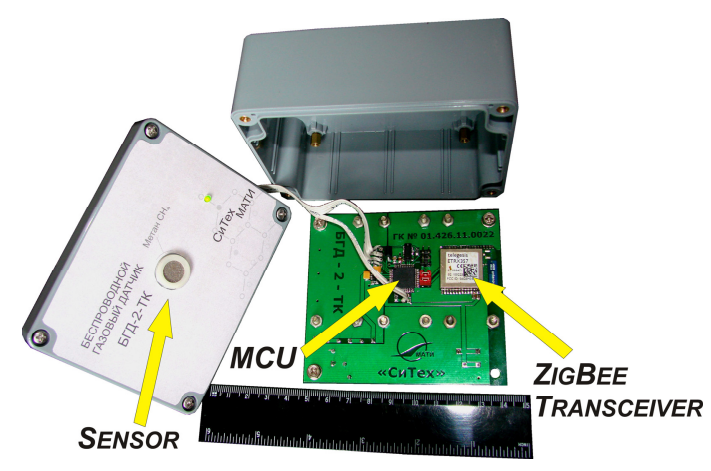

a)

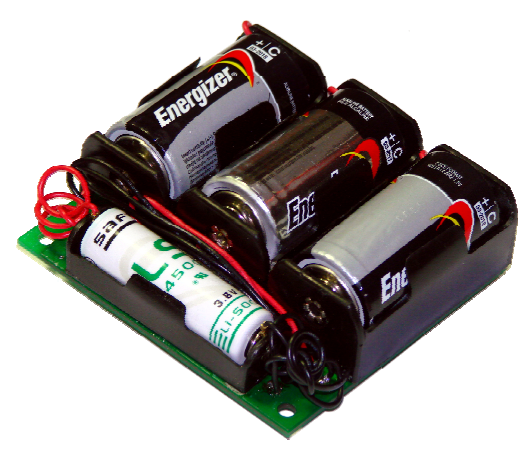

b)

Fig. 9. The hardware prototype of the wireless gas sensor node: (a) top view showing the MCU, the ZigBee module, and the gas sensor, (b) bottom view showing dual power supply: the AA battery for digital section and the $3 \mathrm{xC}$ batteries for analogue section.

Experimental transmission range of the node inside reinforced-concrete buildings is about 20-25 m. It corresponds with the ETRX357 characteristics of $+3 \mathrm{dBm}$ output 
power and $-100 \mathrm{dBm}$ sensitivity. If necessary, the node can be equipped with a compatible long-range ETRX35x-LRS series transceiver, which has $+20 \mathrm{dBm}$ output power and $-106 \mathrm{dBm}$ sensitivity, however it adversely affects energy-saving.

\section{WSN Operation}

In the two previous sections we have demonstrated how to achieve low power consumption of the sensor node by:

- transitioning from a traditional catalytic bead sensors to a planar sensor design;

- combination of the MEMS technology for alumina thin films with the nanotechnology for catalyst materials;

- transitioning from the Wheatstone bridge circuit measurement to a single sensor based measurement (the differential method);

- transitioning from the sensor operation in continuous regime to a pulse regime.

In this section we describe the remaining units of the WSN and explain the principles of WSN operation.

\subsection{Network Coordinator and Wireless Actuator}

The network coordinator (see Figure 10) is based on the LPC2478 microcontroller and equipped with a TFT display to indicate of the sensor node status, an MP3 decoder for gas alarm, and an SD memory card which stores service information and performs the data logging from the sensor nodes. Besides, the network coordinator includes a GSM/GPRS modem MTSMC-G-F4-IP and an Ethernet module for a special service and responsible person alert. Depending on an application demands, the coordinator can be supplied by batteries or a $5 \mathrm{~V} / 550 \mathrm{~mA}$ power adapter.

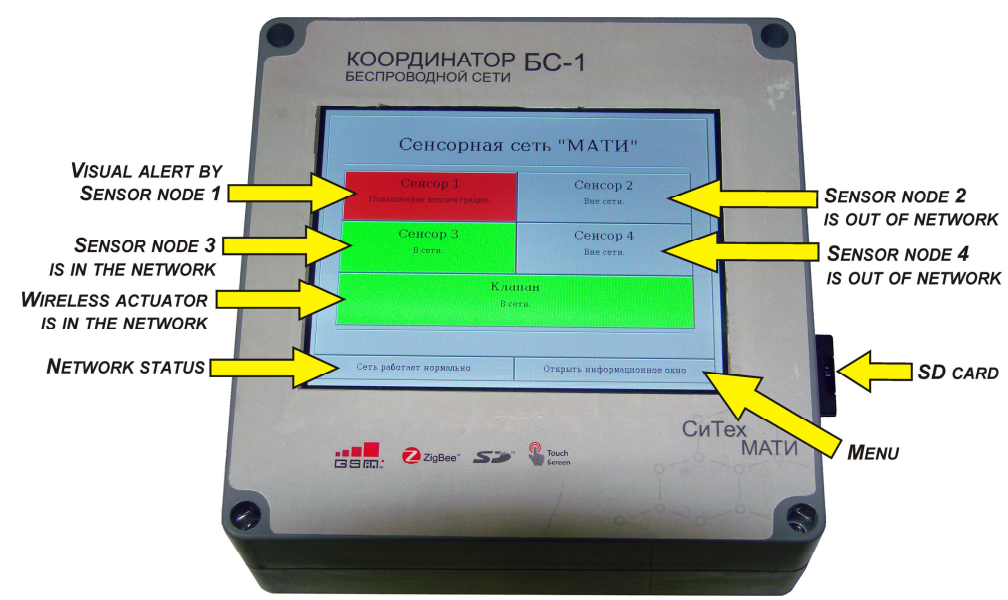

Fig. 10. The photograph of network coordinator where the highlighted sections on the display show the status of the WSN, the sensor nodes, and the wireless actuator(s) 
The status of a sensor node is indicated on the TFT display (with Touch Screen option) of the network coordinator. When a received message contains a gas alert, the coordinator generates a visual alert as well as activates a wireless actuator shown in Figure 11 .

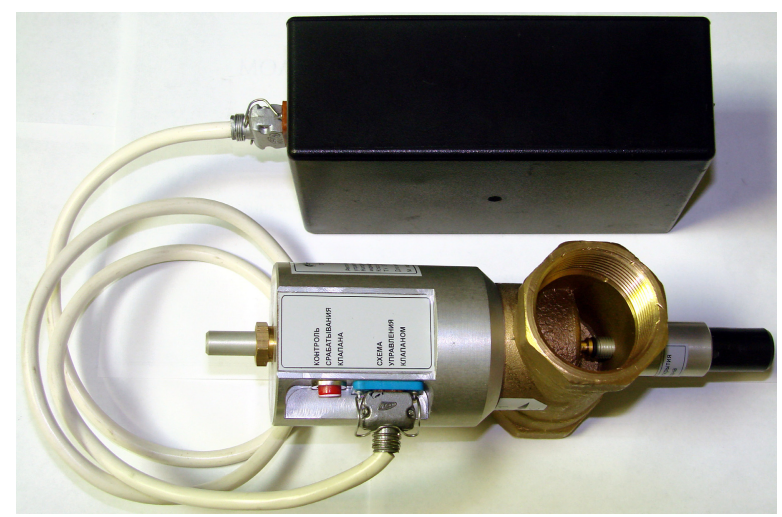

Fig. 11. Physical implementation of the wireless actuator: valve and transceiver with power supply in the housing

In order to prevent disasters caused by the gas leak, the wireless actuators control a valve which locks the gas emission source. The wireless actuator consists of a TGETRX3 transceiver, a power supply, and a valve. The WSN software is designed so that the commands to the wireless actuators can be sent by the sensor and relay nodes as well as by the network coordinator. The units which are located within one hop from the actuator can counteract the leakage rapidly.

\subsection{Gas Detection and Alerting}

The sensor nodes are setup for methane detection within two thresholds. The lower specified threshold is set to $0.15 \% \div 0.5 \%$ vol.; if the concentration rises above this threshold, the WSN will send an alert about the presence of methane in the environment. The second limit is set to $0.5 \% \div 1 \%$, vol. The lower limit of methane explosion is $5 \%$ vol. [12]. The sensor calibration is desirable once a year.

One of the potential applications for the developed WSN is hazardous gas detection in buildings. For this application we choose the cluster topology depicted in Figure 12 which provides secure and reliable operation. The network configuration and data routing are performed by the ZigBee module.

The sensor nodes, $S$, measure the gas presence in the building each $30 \mathrm{~s}$ according to the safety standards [12] and compares the obtained value with the two preset thresholds (the sensor nodes are in sleep mode when are not measuring). If the gas is not detected or its concentration is less than $0.15 \%$, the sensor nodes go to sleep mode. However, the relay nodes, $R$, the network coordinator, $C$, and wireless actuators, $A$, are in receive mode all the time. 
If the sensor node has detected a gas concentration within $0.15 \%-0.5 \%$, it activates the transceiver and sends the message with its identification information to the network coordinator via the relay node(s). As soon as the acknowledgement has been received, the transceiver goes to sleep mode.

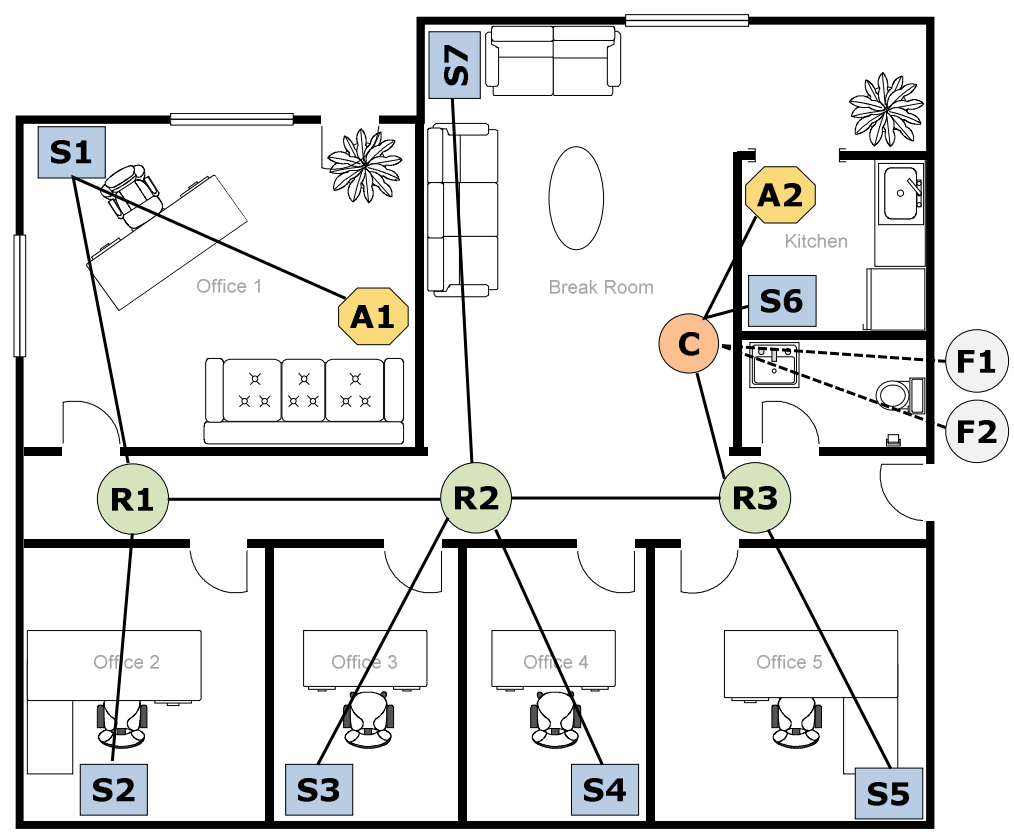

Fig. 12. An example of network topology deployed in an office where $S$ is a sensor node, $C$ is a network coordinator, $A$ is a wireless actuator, $R$ is the relay node, $F 1$ and $F 2$ are the alarm service and the responsible person

The sensor node sends the alert message directly to the wireless actuator if the gas concentration is in the range of $0.5 \%-1 \%$ to close the gas valve. Upon receiving the acknowledgement from the wireless actuator, the sensor node notifies the coordinator.

The network coordinator performs the conversion of the WSN data transmission protocol to other ones for further data transmission. For example, upon receiving an alert message from a sensor node, the coordinator retransmits the alert as SMS to an operator, F2, or alarm service using the on board GSM/GPRS modem. Moreover, the data from a sensor node can be forwarded to a server using the GSM/GPRS modem or an Ethernet module.

The protocol used by the ZigBee specification contains several layers. Apart from the physical and media access control layers described in the IEEE802.15.4 standard, ZigBee provides network, application framework, and application layers. All these layers, except for the application layer, are realized by the firmware of the TGETRX3 transceiver which is installed on each unit of the WSN.

At the application framework layer, the units of the WSN transmit their current status. The status of each sensor node in the WSN is expressed with a unique code which 
consists of three bytes. Data frames contain the information regarding the gas concentration in the environment, power supply status, or errors discovered in the WSN.

\section{Conclusion}

In this work the possibility of developing the sensor node for hazardous gas detetion characterized by low average power consumption $(2.64 \mathrm{~mW}$ per scanning of environment each $30 \mathrm{~s}$ and data communication each 5 minutes) has been for the first time demonstrated. The proposed sensor node is able to operate for up to 641 days using autonomous power supply. It has been designed for atmosphere monitoring as part of a WSN, particularly for methane detection in office buildings, apartments, and industrial premises.

The sensor node low power consumption during environmental scanning has been achieved through the application of special technology (transition from the catalytic bead sensor technology to the planar one), and a careful reading procedure and hardware design. We have proposed a differential method (one sensor required) of gas measurement instead of the widely used Wheatstone bridge (two sensors required) and developed a four-stage pulse heating profile for compensation. The experimental results have shown that the differential method with the planar sensor allows the sensor node to save up to 2.85 times more power, which results in approximately 641 days of operation as opposed to only 225 days in case of the Wheatstone circuit with catalytic bead sensors and to 187 days of state-of-the art systems [2].

In addition, a dual power supply for the sensor node has been implemented. This was done to secure the digital section of the node (MCU and transceiver) from the analogue one (sensor). It prevents the situations where the batteries are getting depleted due to high power consumption of the gas sensor and, therefore, the sensor node becomes remotely uncontrolable.

Due to its reasonable simplicity, wireless connectivity, and low power consumption of the sensor nodes, the designed WSN for hazardous gases detection can be deployed in a short time without entailing considerable maintenance cost. In addition, the use of Ethernet and GSM technologies makes the network easy to manage in realtime.

Acknowledgments. The authors would like to acknowledge the Russian Federal Program "Development of Electronic Components and Radio Electronics" Grant No. 01.426.11.0022. This work was also supported by NATO Collaborative Linkage Grants CBP.CLG.984158.

\section{References}

1. Chong, C.-Y., Kumar, S.P.: Sensor Networks: Evolution, Opportunities, and Challenges. Proceedings of the IEEE 91(8), 1247-1256 (2003)

2. Somov, A., Baranov, A., Savkin, A., Spirjakin, D., Spirjakin, A., Passerone, R.: Development of Wireless Sensor Network for Combustible Gas Monitoring. Sensors and Actuators A: Physical 171(2), 398-405 (2011) 
3. Shnayder, V., Hempstead, M., Chen, B., Allen, G.W., Welsh, M.: Simulating the Power Consumption of Large-scale Sensor Network Application. In: 2nd Conference of Embedded Networked Sensor Systems, Baltimore, Maryland, USA, pp. 188-200 (2004)

4. Ho, S., Koushanfar, F., Kosterev, A., Tittel, F.: LaserSPECks: Laser Spectroscopic TraceGas Sensor Networks - Sensor Integration and Application. In: 6th International Symposium on Information Processing in Sensor Networks, Cambridge, Massachusetts, USA, pp. 226-235 (2007)

5. Xiaoqiang, Z., Zuhou, Z.: Development of Remote Waste Gas Monitor System. In: International Conference on Measuring Technology and Mechatronics Automation, Changsha, China, pp. 1105-1108 (2010)

6. Nakano, S., Yokosawa, K., Goto, Y., Tsukada, K.: Hydrogen Gas Detection System Prototype with Wireless Sensor Networks. In: 4th Conference on Sensors, Irvine, CA, USA, pp. 159-162 (2005)

7. Wobscholl, D.: A Wireless Gas Monitor with IEEE 1451 Protocol. In: The IEEE Sensors Application Symposium, Houston, Texas, USA, pp. 162-164 (2006)

8. Gastronics. True Wireless Gas Detector, http://www.gastronics.com

9. RAE Systems. QRAE II Gas Detector, http: / / www . raesystems . com

10. Hayes, J., Beirne, S., Lau, K.-T., Diamond, D.: Evaluation of a low cost wireless chemical sensor network for environmental monitoring. In: 7th Conference on Sensors, Lecce, Italy, pp. 530-533 (2008)

11. Kim, Y.W., Lee, S.J., Kim, G.H., Jeon, G.J.: Wireless Electronic Nose Network for RealTime Gas Monitoring System. In: International Workshop on Robotic and Sensors Environments, Lecco, Italy, pp. 169-172 (2009)

12. Standard EN 50194:2000: Electrical Apparatus for the Detection of Combustible Gases in Domestic Premises. Test methods and performance requirements (2000)

13. Somov, A., Spirjakin, D., Ivanov, M., Khromushin, I., Passerone, R., Baranov, A., Savkin, A.: Combustible Gases and Early Fire Detection: an Autonomous System for Wireless Sensor Networks. In: 1st International Conference on Energy-Efficient Computing and Networking, Passau, Germany, pp. 85-93 (2010)

14. Somov, A., Ivanov, M., Baranov, A., Savkin, A., Kandeepan, S.: Energy Efficient Tradeoff between Communication and Sensing in Wireless Gas Sensor Node. In: 1st International Workshop on Cognitive Radio and Cooperative Strategies for Power Saving, Lisbon, Portugal, pp. 1-11 (2010) 